\title{
A matéria escura no universo - uma sequência didática para o ensino médio
}

\author{
The dark matter in the universe - a didactic sequence for high school \\ Thiago de C. Gusmão*1, Julia de A. Valente ${ }^{2}$, S. B. Duarte ${ }^{3}$ \\ ${ }^{1}$ Universidade Federal do Estado do Rio de Janeiro, Rio de Janeiro, RJ, Brasil \\ ${ }^{2}$ Colégio Pedro II, Rio de Janeiro, RJ, Brasil \\ ${ }^{3}$ Centro Brasileiro de Pesquisas Física, Rio de Janeiro, RJ, Brasil
}

Recebido em 12 de Março, 2017. Revisado em 15 de Abril, 2017. Aceito em 15 de Abril, 2017.

\begin{abstract}
Nós apresentamos uma proposta pedagógica para introduzir o tema da matéria escura no universo para alunos do ensino médio. O apelo intrínseco deste tema é usado para discutir aplicações de movimento de sistemas planetários e estrelas em galáxias e as relações entre as diferentes formas de energia no movimento desses e outros objetos astronômicos. O principal objetivo é motivar os alunos com ações interativas, incluindo atividades dentro e fora da sala de aula, para aprofundamento do tema. Finalmente, a sequência didática é fechada com uma demonstração numérica do teorema virial gravitacional, levando os alunos a discussões sobre a existência de matéria escura em torno das galáxias, com base no resultado obtido através da verificação do teorema virial gravitacional.

Palavras-chave: Educação em física, teorema do virial, matéria escura
\end{abstract}

We present a pedagogical proposal to introduce the theme of dark matter in the universe to high school students The intrinsic appeal of this subject is used to discuss some applications of movement of planetary system and stars in galaxies and relations between different forms of energy in the movement of stars and others astronomical objects. The main goal is to motivate students with interactive actions, including activities inside and out-classroom, to a further development of the theme. Finally, the didactic sequence is closed with a numerical demonstration of gravitational virial theorem, leading the students to discussions about the existence of dark matter around galaxies, based on the obtained result in verifying the gravitational virial theorem.

Keywords: Physics education, Virial theorem, Dark matter

\section{Introdução}

O estudo de Astronomia recebeu particular destaque nos Parâmetros Curriculares Nacionais do Ensino Médio no Brasil, acreditamos que um dos principais motivos seja a necessidade de prover conteúdos para alicerçar informações que são trazidas por diferentes fontes midiáticas a que os alunos estão expostos em nossos dias. Isto é observado por muitos professores atuantes no ensino médio, como apontado por Cláudio A. Dias em seu livro[1], no qual mostra a necessidade de uma maior ênfase sobre os conhecimentos astronômicos e astrofísicos neste nível de formação. Em sua publicação ele ressalta este ponto através do comentário "Os alunos estão concluindo este nível de ensino sem conhecimento de vários temas na área de Astronomia, que são obrigatórios nos Parâmetros Curriculares Nacionais (PCN's)".

Aproveitando este destaque, vale realçar que seguramente os mais importantes conceitos a serem consolidados no aprendizado do ensino médio são as leis de conservação e as formas de interações entre partículas e corpos manifestas na natureza. Mostrar que estas leis e

*Endereço de correspondência: thiagocq8@yahoo.com.br interações têm suas validades consagradas em diferentes escalas, sem dúvida desperta nos estudantes o fascínio pelo caráter de universalidade das teorias da Física, servindo de instrumento para exploração de fenômenos desde a escala atômica e subatômica até a escala cosmológica. Com esta motivação, apresentamos neste trabalho a utilização da dinâmica do movimento dos corpos sob a ação da interação gravitacional, explicitando as leis de conservação em sistemas fechados. Propomos a análise da distribuição da energia do sistema na sua forma cinética e potencial gravitacional ao longo do tempo, por meio do acompanhamento do cálculo de orbitas planetárias fechadas. Então, evoluímos para a determinação dos valores médios destas grandezas. Desta forma é feita uma verificação do teorema viral mecânico para gravitação através de uma análise numérica da evolução temporal do sistema.

O processo de aquisição de conhecimento envolve o uso de analogias e modelos abstraídos de situações mais familiares[2]. Dentro deste ponto de vista, antes de discutir a dinâmica de objetos massivos dentro de galáxias e de aglomerados galácticos, começamos a abordagem do tema da matéria escura com a análise do virial mecânico 
no sistema planetário solar - afinal, este seria um sistema de objetos celestes bem mais familiares aos alunos.

Na próxima seção é apresentada uma colocação pedagógica para a introdução do tema com base na proposta do Plano Curricular Nacional (PCN) para o ensino médio. $\mathrm{Na}$ seguinte, são desenvolvidas as técnicas, metodologia e instrumentos de cálculo utilizados na proposta e seus resultados são expostos para a exploração da relação do virial como elemento básico para argumentação da existência da matéria escura no Universo. Incluímos como suplemento do artigo o apêndice A, com instruções para uso dos recursos computacionais utilizados para a elaboração de cálculos e gráficos, a fim de auxiliar professores e alunos não familiarizados com estas ferramentas. Como material suplementar também acrescentamos o apêndice B apresentando os resultados da avaliação da aplicação da sequência didática elaborada neste trabalho.

\section{Astronomia e temas cosmológicos atuais no PCN}

Um dos temas propostos para discussão no ensino básico é a Matéria Escura que, na literatura científica, aparece como um problema em aberto. Também é colocado como um dos grandes desafios à compreensão de nosso Universo.

É requisito do $\mathrm{PCN}$, do ensino médio - Ciências da Natureza na área de Física -, o efetivo aprendizado do tema estruturador Universo, Terra e Vida, que é composto das seguintes unidades temáticas: Compreensão de aspectos da evolução dos modelos da ciência para explicar a constituição do Universo (matéria, radiação e interações) através dos tempos, identificando especificidades do modelo atual.[1]

O presente trabalho introduz uma proposta de atividade interativa a ser realizada em sala de aula e/ou em laboratório de informática, para discussão sobre a detecção da matéria escura a partir do teorema Virial. O conceito de matéria escura aparece como uma necessidade de solucionar a inconsistência entre as observações e as previsões de velocidades de estrelas ao redor do centro galáctico com base no conteúdo de matéria luminosa da galáxia. Este fato levou pesquisadores a supor a existência de alguma forma de matéria não observada, mas gravitacionalmente ativa, influenciando o movimento das estrelas na galáxia. Na imagem da figura 1 temos um quadro artístico mostrando a relação existente de matéria escura na Via Láctea.

Com o auxílio de tabelas, cálculos e gráficos, utilizando o software Exce $]^{1}$ podemos estudar inicialmente o movimento de um corpo celeste (planetas, por exemplo) em torno do Sol. Considerando que o corpo realiza uma órbita fechada, é possível calcular a energia cinética e potencial em cada ponto da trajetória do planeta e as-

${ }^{1}$ Escolhemos este software por ser de grande difusão e com possibilidade de maior familiaridade dos alunos com o mesmo. Além da grande facilidade de cálculos e recursos gráficos que disponibiliza.

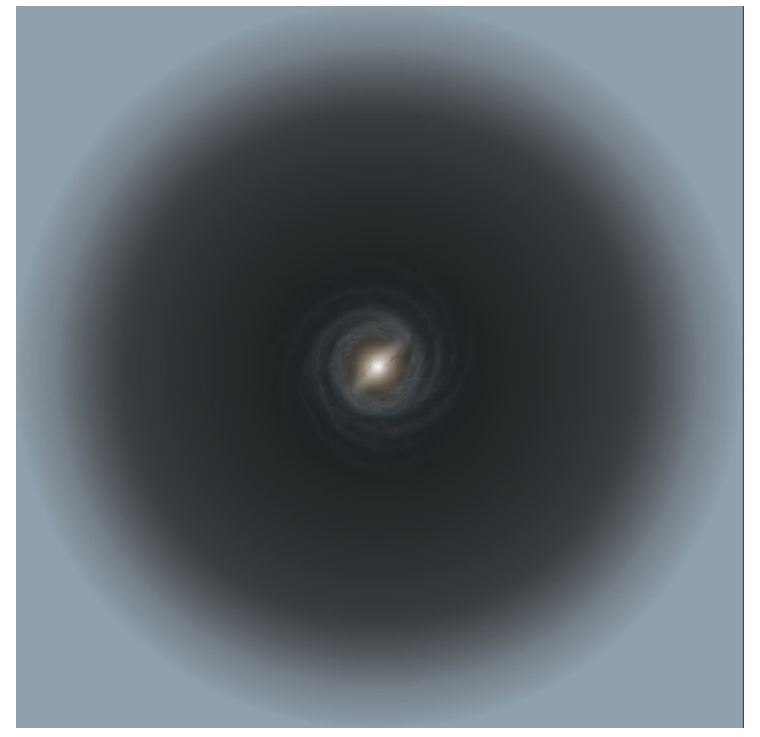

Figura 1: Uma galáxia no centro da figura e toda a Matéria escura existente em torno dela (parte escura).

sim obter uma média dessas energias. Com a exploração numérica deste movimento é possível verificar o teorema virial, que estabelece a relação entre a energia cinética e potencial, em termos de média temporal, de uma órbita planetária em torno do Sol.

A partir dessa experiência, podemos generalizar a análise para dinâmica de uma galáxia: uma estrela que gira em torno do centro galáctico em uma órbita fechada poderia ser tratada de forma análoga ao que foi desenvolvido anteriormente para o movimento dos planetas. Quando aplicado aos corpos celestes mais complexos (galáxias ou aglomerados de galáxias) é observado que, para o teorema ser satisfeito, é necessário supor a existência de alguma forma de matéria gravitacionalmente ativa e não visível - o que se convencionou chamar de "Matéria Escura". A figura 2 ilustra a proporção da matéria escura, energia escura e matéria visível contida no universo. O setor escuro evidencia o grande desconhecimento reinante sobre composição e estrutura do Universo, desafiando as gerações de futuros pesquisadores. A maior proporção é de energia escura, concebida para se explicar a expansão inesperada do universo. Esta se diferencia da matéria escura e de outra forma qualquer de radiação ou matéria conhecida por apresentar uma pressão negativa. Somente uma proporção como a indicada na figura poderia justificar o comportamento hoje observado para a expansão do universo. A proposta aqui apresentada refere-se à exploração apenas da questão da descoberta da matéria escura na composição do universo.

\section{Metodologia e instrumentos didáticos para exploração do tema}

O público alvo dessa atividade são alunos do ensino básico que cursam o ensino médio. É pré-requisito para 


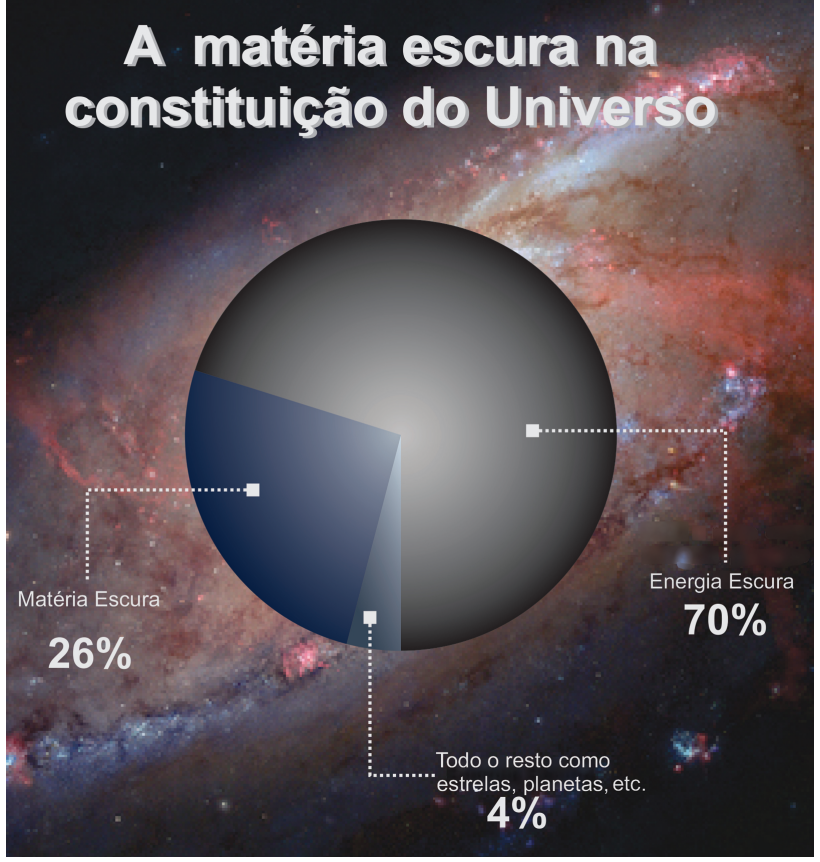

Figura 2: A distribuição da Matéria Escura, Energia Escura e matéria visível no Universo.

discussão o estudo de movimento dos corpos (MU e MUV), que serão utilizados na elaboração da proposta, composta de três momentos colocados pelos professores para atividades com suas turmas.

No primeiro momento é realizada uma visita ao Museu de Astronomia e Ciências Afins, ou Planetários, onde os alunos possam conhecer espaços expositivos relacionados com o tema da atividade, como por exemplo, fenômenos astronômicos (estações do ano, fases da Lua e eclipses), corpos celestes (nebulosas, aglomerados e galáxias). Nesse momento, uma das atividades mais recomendadas é a observação do céu em telescópios. Essa primeira etapa é de grande importância para motivação e envolvimento dos alunos nas etapas futuras.

$\mathrm{Na}$ dificuldade de realizar essa etapa é importante que seja substituída por outra atividade que promova nos estudantes uma inserção no contexto da temática, por exemplo: vídeos, palestras ou atividades práticas.

"Os PCNEM recomendam também que a apropriação dos conhecimentos físicos deve ser desenvolvida passo a passo, a partir dos elementos próximos, práticos e vivenciais do aluno, reconhecendo-se a necessidade de que o abstrato seja construído "concretamente", a partir de situações reais, sem que se faça apelo a definições dogmáticas ou a tratamentos impropriamente triviais. Sugerem, igualmente que e essencial trabalhar com modelos, introduzindo-se a própria ideia de modelo através da discussão de modelos microscópicos e que tais modelos devem ser construídos a partir da necessidade explicativa dos fatos em correlação direta com os fenômenos macroscópicos que se quer explicar. [3]"
É importante que nesse momento valorize-se o protagonismo dos alunos e sua tomada de decisão na hora de escolher os espaços e atividades a serem realizadas.

O segundo momento é realizado no colégio e nesta ocasião revisamos conceitos de movimento dos corpos que são importantes para o entendimento do que será realizado. Também são apresentadas grandezas como energia cinética e potencial. A partir daí começaremos uma discussão sobre o movimento de um planeta em torno do Sol, primeiramente para uma órbita circular, analisando de forma analítica as forças envolvidas (centrípeta $=$ gravitacional) e a cinemática do movimento. A fim de facilitar o acompanhamento, por parte dos alunos, da evolução das grandezas envolvidas, utilizaremos os recursos gráficos do software para a exposição rápida dos resultados e manipulação destes. Dessa maneira conseguimos mostrar a forma da trajetória, as variações dos componentes de velocidade nos eixos x, y do plano da orbita. Além disso, pode-se explorar a energia cinética e potencial durante um ciclo do movimento em torno do Sol. Nesse momento é importante mencionar alguns detalhes na elaboração/construção da planilha.

As linhas da planilha são alimentadas com informações da grandeza em questão em cada coluna. As células de uma mesma linha correspondem aos valores destas grandezas num dado instante de tempo. A figura 3 mostra uma cópia da tela do computador exibindo a planilha com as grandezas e seus valores. Chamamos atenção para o fato de estarmos construindo a órbita com passos muito pequenos no tempo. A ideia é aproximarmos o movimento do planeta durante este pequeno passo por um movimento retilíneo uniformemente variado (MUV). Observe que por esse motivo devemos ter um número de passos muito grande cobrindo toda orbita do planeta. Imaginem por exemplo algo como utilizar 10.000 passos para que o gráfico da órbita esteja compatível com a realidade.

Outra análise interessante é a mudança no valor da velocidade inicial para visualizar os diferentes tipos de órbitas descritas pelo planeta. Partindo do estudo numérico de uma órbita circular, ampliaremos o estudo para órbitas elípticas e parabólicas. Pode-se plotar a trajetória, fazer gráficos de velocidades no tempo e melhor analisar diferenças entre os diversos tipos de órbitas. Finalizamos analisando a razão entre a média dos valores da energia potencial e a media da energia cinética em cada instante.

Pela órbita circular apresentar-se como um movimento mais familiar aos estudantes, para que adquiram habilidade com os recursos computacionais necessários, o estudo é iniciado por ela. No apêndice A e nos vídeos ali inseridos consta o procedimento passo a passo para obtenção dos resultados apresentados na planilha da figura 3. As colunas desta planilha mostram as grandezas relevantes para o estudo desta órbita e com estes resultados podemos elaborar gráfico para a evolução no tempo destas grandezas. 


\begin{tabular}{|c|c|c|c|c|c|c|c|c|c|c|c|c|c|c|c|c|}
\hline & A & B & C & D & E & $\mathrm{F}$ & G & $\mathrm{H}$ & I & J & K & L & M & $\mathrm{N}$ & 0 & $\mathbf{P}$ \\
\hline 1 & $X(\mathrm{~m})$ & $Y(m)$ & $\operatorname{Ax}\left(\mathrm{m} / \mathrm{s}^{2}\right)$ & $\operatorname{Ay}\left(\mathrm{m} / \mathrm{s}^{2}\right)$ & $V_{x}(\mathrm{~m} / \mathrm{s})$ & $V y(m / s)$ & $V(m / s)$ & $\operatorname{Epg}(J)$ & $\operatorname{Ec}(J)$ & $\Delta t(s)$ & G & Sol (Kg) & Terra(Kg) & tempo & $\mathrm{Epg} / \mathrm{Ec}$ & Vel. Inicial \\
\hline 2 & $1,5 E+11$ & $0,00 E+00$ & $-0,00587$ & 0 & 0 & $2,97 E+04$ & $2,97 E+04$ & $-5,3 E+33$ & $2,63 E+33$ & 3150 & $6,67 E-11$ & $1,98 \mathrm{E}+30$ & $5,97 E+24$ & $0,00 E+00$ & $-2,00 E+00$ & 29672,21 \\
\hline 3 & $1,5 E+11$ & $9,35 E+07$ & $-0,00587$ & $-3,7 E-06$ & $-18,4892$ & $2,97 E+04$ & $2,97 \mathrm{E}+04$ & $-5,3 E+33$ & $2,63 E+33$ & 3150 & $6,67 E-11$ & $1,98 \mathrm{E}+30$ & $5,97 E+24$ & $3,15 \mathrm{E}+03$ & $-2,00 E+00$ & \\
\hline 4 & $1,5 E+11$ & $1,87 E+08$ & $-0,00587$ & $-7,3 \mathrm{E}-06$ & $-36,9843$ & $2,97 E+04$ & $2,97 E+04$ & $-5,3 E+33$ & $2,63 E+33$ & 3151 & $6,67 \mathrm{E}-11$ & $1,98 \mathrm{E}+30$ & $5,97 E+24$ & $6,30 E+03$ & $-2,00 E+00$ & \\
\hline 5 & $1,5 \mathrm{E}+11$ & $2,80 \mathrm{E}+08$ & $-0,00587$ & $-1,1 \mathrm{E}-05$ & $-55,4853$ & $2,97 E+04$ & $2,97 E+04$ & $-5,3 E+33$ & $2,63 E+33$ & 3152 & $6,67 \mathrm{E}-11$ & $1,98 E+30$ & $5,97 \mathrm{E}+24$ & $9,45 E+03$ & $-2,00 E+00$ & \\
\hline 6 & $1,5 E+11$ & $3,74 E+08$ & $-0,00587$ & $-1,5 \mathrm{E}-05$ & $-73,9921$ & $2,97 E+04$ & $2,97 E+04$ & $-5,3 E+33$ & $2,63 E+33$ & 3153 & $6,67 \mathrm{E}-11$ & $1,98 \mathrm{E}+30$ & $5,97 E+24$ & $1,26 \mathrm{E}+04$ & $-2,00 E+00$ & \\
\hline 7 & $1,5 \mathrm{E}+11$ & $4,68 E+08$ & $-0,00587$ & $-1,8 \mathrm{E}-05$ & $-92,5047$ & $2,97 E+04$ & $2,97 \mathrm{E}+04$ & $-5,3 E+33$ & $2,63 E+33$ & 3154 & $6,67 E-11$ & $1,98 \mathrm{E}+30$ & $5,97 E+24$ & $1,58 \mathrm{E}+04$ & $-2,00 E+00$ & \\
\hline 8 & $1,5 E+11$ & $5,61 E+08$ & $-0,00587$ & $-2,2 \mathrm{E}-05$ & $-111,023$ & $2,97 E+04$ & $2,97 E+04$ & $-5,3 E+33$ & $2,63 E+33$ & 3155 & $6,67 \mathrm{E}-11$ & $1,98 \mathrm{E}+30$ & $5,97 E+24$ & $1,89 \mathrm{E}+04$ & $-2,00 E+00$ & \\
\hline 9 & $1,5 E+11$ & $6,55 E+08$ & $-0,00587$ & $-2,6 \mathrm{E}-05$ & $-129,547$ & $2,97 E+04$ & $2,97 E+04$ & $-5,3 E+33$ & $2,63 E+33$ & 3156 & $6,67 \mathrm{E}-11$ & $1,98 \mathrm{E}+30$ & $5,97 E+24$ & $2,21 E+04$ & $-2,00 E+00$ & \\
\hline 10 & $1,5 E+11$ & $7,49 \mathrm{E}+08$ & $-0,00587$ & $-2,9 \mathrm{E}-05$ & $-148,078$ & $2,97 E+04$ & $2,97 \mathrm{E}+04$ & $-5,3 E+33$ & $2,63 E+33$ & 3157 & $6,67 E-11$ & $1,98 \mathrm{E}+30$ & $5,97 E+24$ & $2,52 E+04$ & $-2,00 E+00$ & \\
\hline
\end{tabular}

Figura 3: Recorte da tela do computador mostrando parte de uma planilha Excel com cálculos obtidos para os valores das grandezas especificadas na tabela 1 , para o caso de uma orbita circular. Seu preenchimento e detalhes do calculo estão indicados passo a passo no Apêndice A.

Tabela 1: Especificação das grandezas envolvidas no cálculo. Na parte superior são especificadas as condições iniciais para a determinação numérica das orbitas planetárias a serem estudadas. Na primeira coluna da tabela estão as abreviações das grandezas utilizadas. Na coluna dois as grandezas são nomeadas e na três estão instruções ou expressões que servem para orientação no preenchimento das células na planilha Excel (vide apêndice). Atenção na escolha do valor do intervalo de tempo (passo), para obter uma órbita fechada (figura 5).

\begin{tabular}{|c|c|c|}
\hline \multicolumn{3}{|c|}{$\begin{array}{l}\text { Condição inicial a ser adicionada na primeira linha da planilha: } \\
\mathrm{X} 0=\text { Raio médio da órbita do planeta }=(\mathrm{R} \text { afélio }+\mathrm{R} \text { periélio }) / 2 \\
\mathrm{~V} 0 \mathrm{x}=0 ; \mathrm{Y} 0=0 ; \mathrm{V} 0 \mathrm{y}=\text { livre }\end{array}$} \\
\hline $\mathrm{X}$ & Abscissa da posição do planeta. & $\frac{1}{2} \mathrm{a}_{\mathrm{x}} \Delta \mathrm{t}^{2}+\mathrm{v}_{0 \mathrm{x}} \Delta \mathrm{t}+\mathrm{x}_{0}$ \\
\hline $\mathrm{Y}$ & Ordenada da posição do planeta & $\frac{1}{2} \mathrm{a}_{\mathrm{y}} \Delta \mathrm{t}^{2}+\mathrm{v}_{0 \mathrm{y}} \Delta \mathrm{t}+\mathrm{y}_{0}$ \\
\hline$a_{x}$ & Aceleração do planeta no eixo-x & $\frac{G M_{\text {sol }} X}{\left(X^{2}+Y^{2}\right)^{3 / 2}}$ \\
\hline$a_{y}$ & Aceleração do planeta no eixo-y & $\frac{G M_{s o l} Y}{\left(X^{2}+Y^{2}\right)^{3 / 2}}$ \\
\hline $\mathrm{V}_{\mathrm{x}}$ & Velocidade no eixo-x & $V_{x}+a_{x} \Delta t$ \\
\hline $\mathrm{V}_{\mathrm{y}}$ & Velocidade no eixo-y & $V_{y}+a_{y} \Delta t$ \\
\hline $\mathrm{V}$ & Modulo da velocidade & $\left(V_{x}^{2}+V_{y}^{2}\right)^{1 / 2}$ \\
\hline $\mathrm{E}_{\mathrm{pot}}$ & Energia potencial & $\frac{-G M_{s o l} m}{\left(X^{2}+Y^{2}\right)^{1 / 2}}$ \\
\hline $\mathrm{E}_{\mathrm{c}}$ & Energia cinética & $\frac{m V^{2}}{2}$ \\
\hline$\Delta \mathrm{T}$ & Intervalo de tempo considerado & $\begin{array}{l}\text { Fazer escolha adequada com o aluno } \\
\text { (vide discussão no texto) }\end{array}$ \\
\hline G & Constante gravitacional. & Entrar com dados em unidade adequada \\
\hline $\mathrm{M}_{\text {Sol }}$ & Massa do Sol & Entrar com dado observacional \\
\hline $\mathrm{m}$ & Massa do planeta. & Entrar com dado observacional \\
\hline Tempo & Tempo decorrido. & Entrar com dado observacional \\
\hline$\frac{E p o t}{E_{c}}$ & Relação do Virial. & Saída como resultado \\
\hline
\end{tabular}

A figura 4 apresenta a trajetória circular obtida com os resultados mostrados no recorte da planilha da figura 3. Neste caso, o objetivo é poder comparar o resultado numérico obtido com a planilha e os resultados analíticos que o professor pode desenvolver em sala com a turma. Serve também para introduzir os recursos gráficos da planilha para os alunos. No gráfico da figura 4, a velocidade inicial está na direção do eixo Y - com valor determinado analiticamente para o caso do movimento circular uniforme da órbita da terra em torno do sol ( o que corresponde a $\mathrm{V}_{\mathrm{y}}=\sqrt{\frac{G \cdot M}{R}}$ ).
É oportuno salientar a questão da precisão dos cálculos efetuados pelo software e sobretudo das aproximações introduzidas no cálculo da trajetória, quando usamos o MUV para evoluí-la num passo temporal. Por exemplo, é crucial a escolha do tamanho deste passo entre posições sucessivas na planilha. Na figura 5 mostramos o que acontece quando escolhemos um passo muito grande, ou seja, um intervalo de tempo que permite que erros se acumulem devido as aproximações demasiadas dos cálculos. Nem mesmo a conservação da energia total do sistema é mantida, levando a uma órbita que espiraliza e 


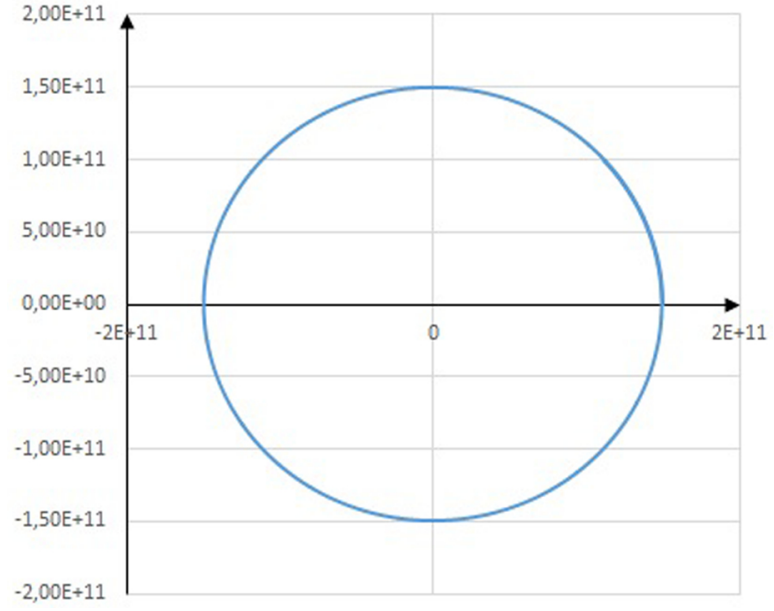

Figura 4: Gráfico correspondente a órbita circular, extraído da planilha do Excel 2007

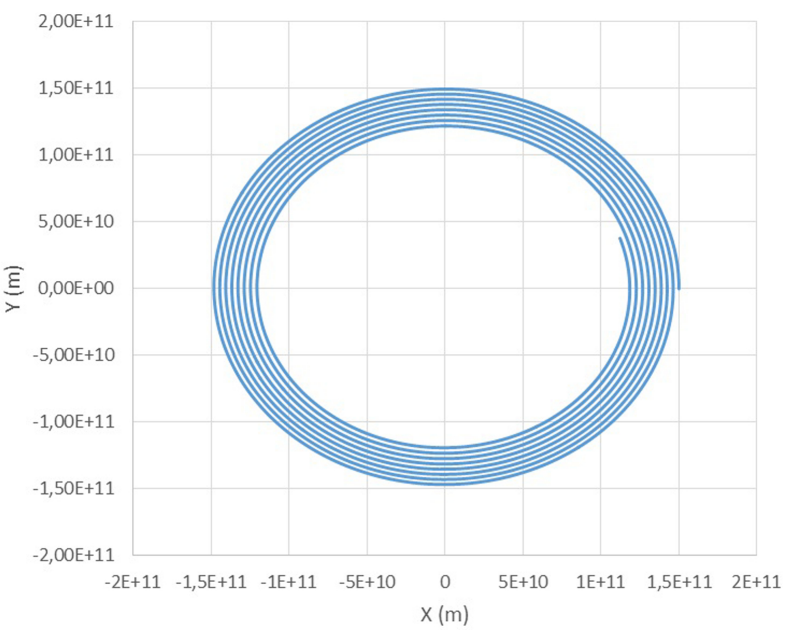

Figura 5: Gráfico correspondente a órbita circular, com o intervalo de tempo "mal escolhido" (grande demais), levando a um acúmulo de erros que não permite o fechamento da órbita.

não se fecha. Vale resaltar que todos os cálculos numéricos (até mesmo aqueles mais precisos no padrão NASA), apresentam esse tipo de defeito, que é aprimorado pela tolerância imposta sobre o erro do resultado, através do controle do passo. Incluimos esta discussão para que no processo interativo professor e alunos possam vivenciar de forma concreta o papel das aproximações matemáticas nas soluções de problemas físicos.

Completando o estudo das órbitas circulares aproveitamos os recursos gráficos da planilha para analisar o comportamento das projeções das velocidades e das acelerações nos eixos X e Y. A figura 6 deve ser apresentada evidenciando o caráter senoidal e cosenoidal da evolução temporal destas grandezas.

Ainda no caso da trajetória circular é importante salientar, para os nosso propósitos em verificar o teorema virial, que a razão entre a energia potencial e cinética é constante e igual a - 2. Isto não acontece quando a órbita torna-se eliptica, o que pode ser facilmente mos-
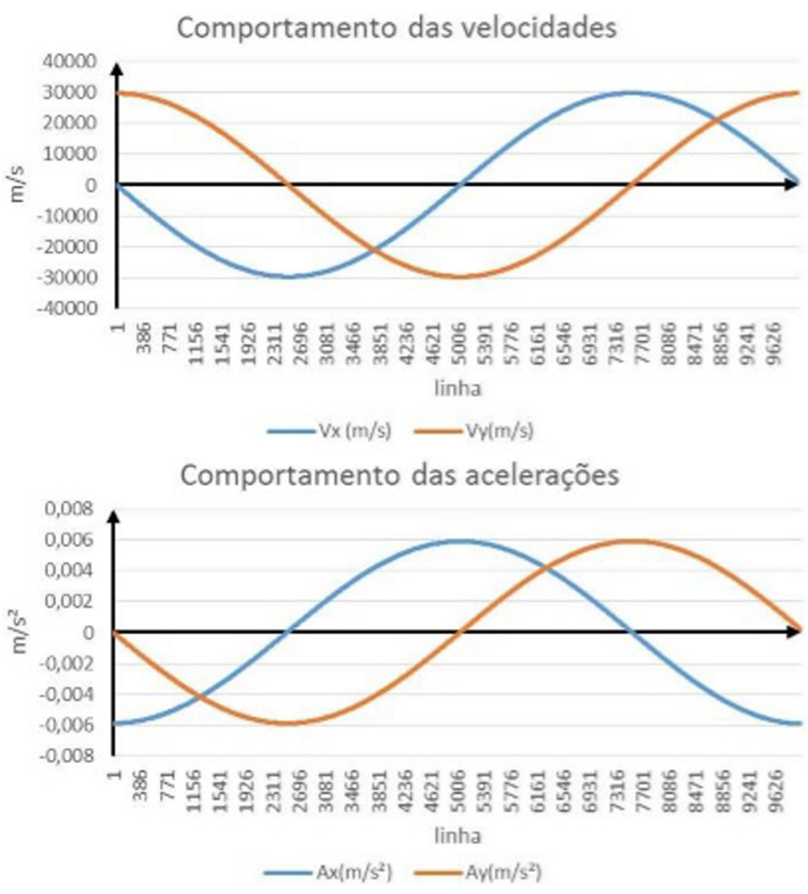

Figura 6: Gráfico da evolução temporal dos componentes das acelerações (em baixo) e dos componentes de velocidade (em cima) correspondente a órbita circular da figura 4. Em azul a componente no eixo-X e em vermelho a componente no eixo-Y.

trado usando como velocidade inicial para o movimento um valor um pouco superior àquele que foi escolhido para o caso das órbitas circulares, $\mathrm{V}_{\mathrm{y}}=\sqrt{\frac{G \cdot M}{R}}$. No caso da figura 7, a órbita será elíptica, o sol ocupará um dos focos e o valor típico da velocidade inicial no eixo-y $V y=\sqrt{\frac{3 G M}{2 R}}$.

Neste caso de órbitas elípticas, a distância ao Sol muda a cada instante e as energias potenciais e cinéticas variam a cada momento. A razão entre estas grandezas também não se mantém constante. Porém, a razão entre as médias

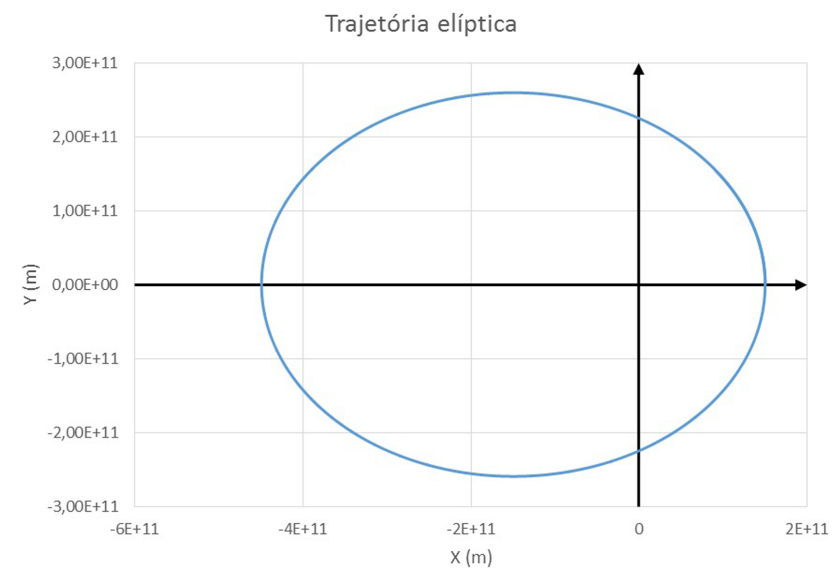

Figura 7: Orbita elíptica com o sol no foco tomado como centro de coordenadas. Obtida com o aumento da velocidade inicial do planeta na direção do eixo-y. 
destas grandezas tomadas dentro do período tem um valor bem especificado e é o que determina o teorema do virial. Os dois gráficos a seguir mostram o comportamento das velocidades e das acelerações nos eixos X e Y na órbita elíptica. Na figura 8, apresentamos a evolução temporal das componentes de velocidade e de aceleração para o caso dessa órbita. Importante salientar as diferenças entre esse caso e a situação analisada para o caso das orbitas circulares da figura 6 .

Quando a velocidade inicial para o movimento do planeta for superior a um dado valor crítico, ele tem energia cinética suficiente para escapar da atração do Sol e a trajetória torna-se uma parábola. Neste caso, ela é aberta - logo, não se aplica o teorema virial. As trajetórias parabólicas começam a ocorrer quando a velocidade inicial corresponde a $\mathrm{V}_{\mathrm{y}}=\sqrt{\frac{2 \cdot \mathrm{G} . \mathrm{M}}{\mathrm{R}}}$ (a velocidade de escape). A razão entre energia cinética e potencial é igual a 1 neste caso. Nessa trajetória específica, a razão se mantém igual em cada ponto. As figuras 9 e 10 mostram a trajetória e o comportamento das componentes de velocidade e de aceleração do movimento do planeta neste caso.

Para finalizar esta seção devemos retomar o aspecto didático da proposta e deixar claro que a metodologia utilizada é apenas um instrumento para alcançarmos o objetivo de passar os conceitos com base em resultados obtidos. Portanto, neste segundo momento devemos cuidar para não incidirmos no que foi mencionado por Borges no artigo [2] quanto à utilização de técnicas e novos métodos didáticos: "o problema com a educação,
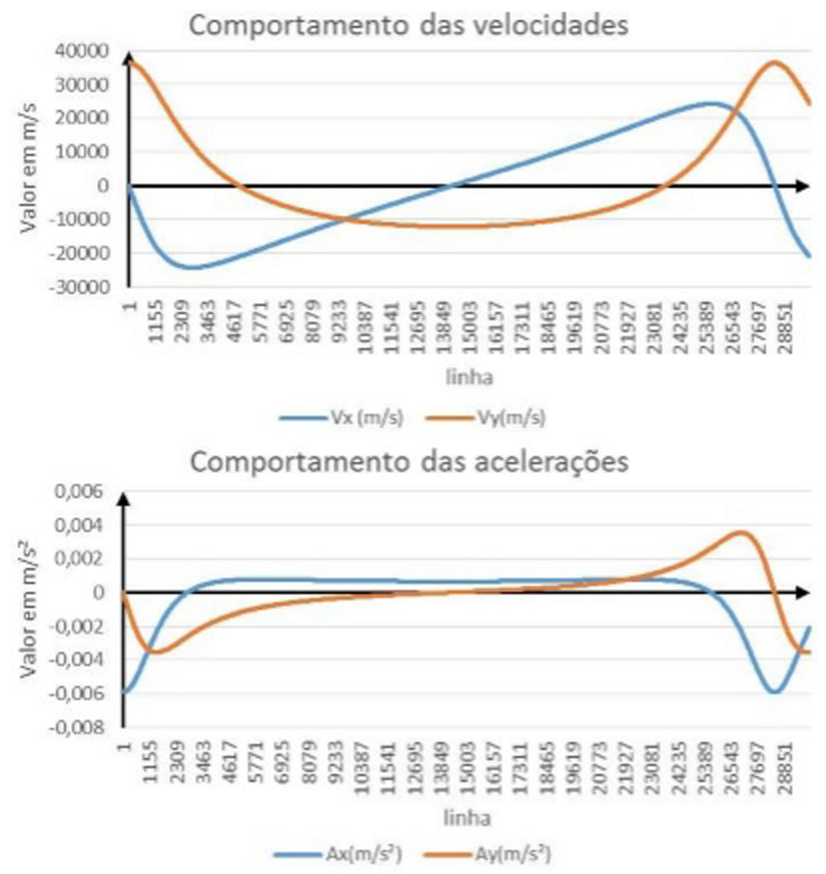

Figura 8: Gráfico da evolução temporal dos componentes das acelerações (em baixo) e dos componentes velocidade de velocidade (em cima), para o caso de uma órbita elíptica. Em azul a componente no eixo-X e em vermelho a componente no eixo-Y. no entanto, não é o método de apresentação é a assimilação do material apresentado. Infelizmente a maioria dos usos da tecnologia em educação foca na apresentação da informação".

A partir da análise dos dados fica clara a relação das energias nos três tipos de órbitas. Podemos então avançar para o terceiro momento da proposta e começar as discussões sobre a generalização dessa abordagem para galáxias ou aglomerados de galáxias. Comentamos que essa verificação não é consistente quando admitimos que esses corpos sejam estabilizados, pois os dados referentes à velocidade tangencial em regiões mais periféricas dessas gigantescas estruturas não correspondem àquela prevista, sendo necessário "adicionar" massa para que

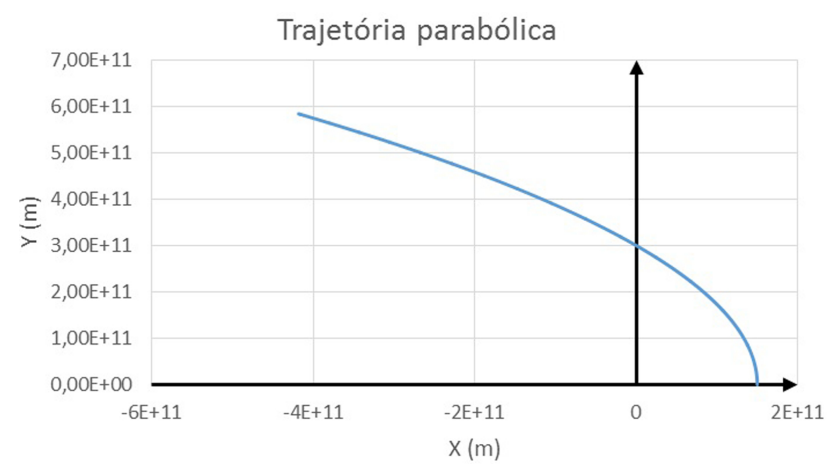

Figura 9: A trajetória do planeta para o caso em que escapa da atração do Sol. Abaixo se mostra o comportamento dos componentes de velocidade e do modulo da velocidade do planeta.
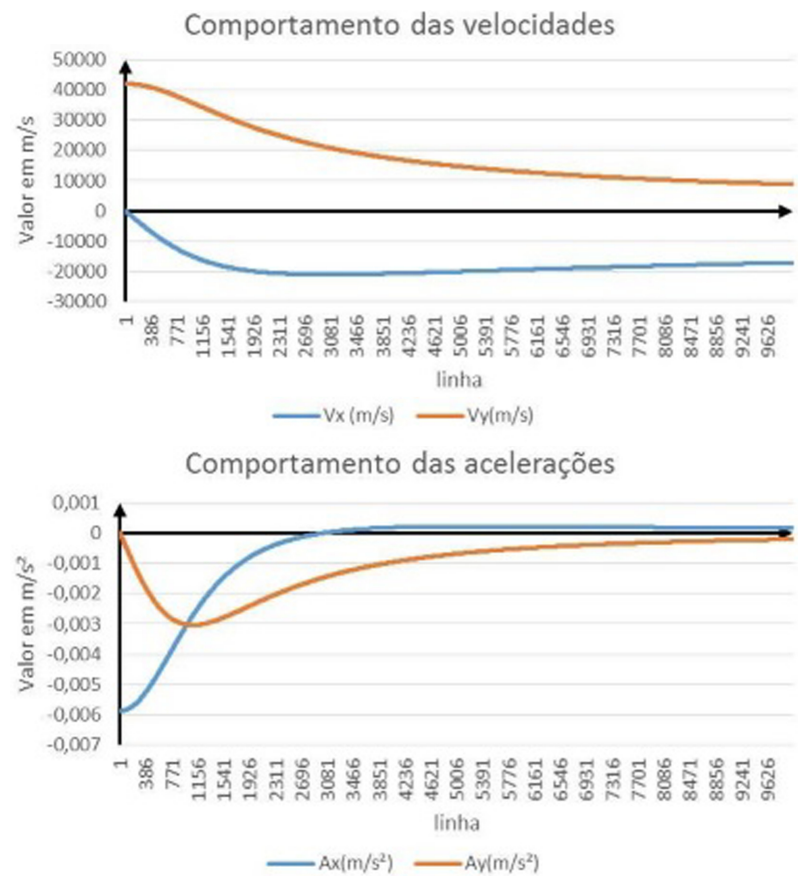

Figura 10: Evolução dos componentes da velocidade e da aceleração para o caso da trajetória parabólica mostrada na figura anterior. Para grandes valores de tempo a aceleração tende a zero, o planeta se afasta muito do sol e a força gravitacional se anula. 
o teorema seja satisfeito. Dessa maneira, mostramos a necessidade da existência da matéria escura permeando toda a galáxia.

\section{O virial e a argumentação da existência de matéria escura}

Este seria o terceiro momento da proposta e o ponto importante no que se refere à conexão das análises das órbitas e da verificação do teorema virial com o tema propriamente dito. Devem ser feitas analogias do estudo da órbita dos planetas com órbitas de astros em grandes estruturas cosmológicas, por exemplo, o movimento da órbita do Sol em torno da nossa galáxia. Analogamente ao que fizemos para a órbita de planetas em torno do sol, podemos supor a veracidade do teorema virial para a órbita das estrelas em torno da galáxia.[4] Assim, a relação entre as energias de cada estrela deveria respeitar o teorema, considerando a galáxia como um sistema equilibrado. Porém, ao analisarmos as estrelas em diferentes órbitas em torno do centro da galáxia, essa relação não é satisfeita. Verificamos que, ao se afastar do centro da galáxia, as estrelas que deveriam diminuir suas velocidades, as mantém constante.

$\mathrm{Na}$ figura 11 podemos perceber o gráfico que indica a relação da velocidade da órbita e a distância da estrela em relação ao centro da galáxia. A parte tracejada indica a relação esperada pelo teorema virial para essas estrelas. Já a linha amarela, o que realmente se observa nessa relação para uma galáxia em espiral, como a Via Láctea. Essa diferença existente entre o que observamos e aquela prevista pelo teorema, nos leva a acreditar na existência de "alguma coisa" de natureza gravitacional, mas que não pode ser observada mediante ondas eletromagnéticas - a essa grandeza misteriosa denominamos Matéria Escura.

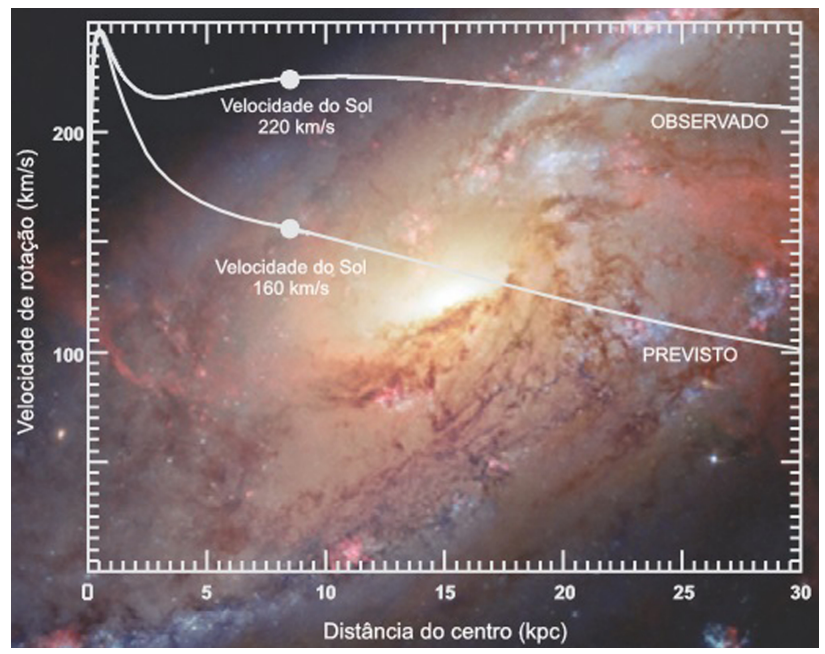

Figura 11: Curva ilustrativa do comportamento da velocidade de rotação de estrelas em torno do centro galácticos, mostrando a diferença dos valores observados e previsto para qualquer galáxia.
O primeiro cientista a mencionar a existência da "Matéria Escura"foi Fritz Zwicky[5], na década de 30. Os primeiros estudos verificaram que toda radiação emitida por um aglomerado de galáxias, tanto na frequência visível quanto em raios-x, não correspondia à estimativa de massa daquele aglomerado. Essa afirmação confirmava aquela feita com a utilização do teorema Virial. Porém essas duas estimativas precisavam admitir um sistema equilibrado, o que em diversos casos causava um desconforto, já que em muitos aglomerados esse equilíbrio poderia não existir. Já na década de 80, após as observações do eclipse solar, que respondeu às questões levantadas pela Relatividade Geral, surgiu uma nova ferramenta as lentes gravitacionais. O uso desse método não estaria aprisionado ao equilíbrio do sistema e mostrou ser uma ferramenta fortíssima para detecção da matéria escura.

Com relação às galáxias, essa evidência também é notória. Em seu artigo "O lado escuro do Universo", Laerte Sodré Jr relata: "No sistema solar, quanto mais distante um planeta estáì do Sol, mais devagar ele o orbita: isso eì denominado movimento kepleriano. Algo parecido era esperado no movimento de rotação das estrelas do disco em relação ao centro das galáxias espirais. Verificou-se, porém, que, em vez da rotação decrescer com a distância ao centro, ela permanecia aproximadamente constante. Esse resultado foi interpretado por Freeman, em 1970, como evidência de que as galáxias continham uma massa não detectada, com uma distribuição diferente das estrelas. Aparentemente, as galáxias são imensos esferoides de matéria escura, com as estrelas e outras formas de matéria bariônica residindo na região central." [6]

Em uma perspectiva mais atual, a matéria escura continua como um ponto de interrogação atormentador e ao mesmo tempo excitante, motivando esforços e inspirando concepções fantásticas, como por exemplo, as Partículas Massivas que interagem fracamente (WIMP, sigla em inglês), formadas por um conjunto de partículas, entre elas, o famoso neutrino com uma massa, segundo Carlos Alexandre Wuensche, aproximadamente um centésimo de milésimo da massa do elétron e com velocidade próxima da luz. O artigo de Wuensche, "A Radiação Cósmica de Fundo em Micro-ondas e a Formação de Estruturas no Universo: Uma Visão Atual"[7] é uma excelente leitura para aqueles mais interessados.

\section{Resultados esperados, comentários e conclusões finais}

O presente trabalho tem como principal objetivo fortalecer e ampliar o universo de atividades a serem realizadas no ensino médio a fim de enriquecer o processo de ensinoaprendizagem relacionada a temáticas de astronomia. A ideia é também introduzir no ensino de astronomia atividades interativas a partir do envolvimento com tecnologias. 
Segundo Borges, "A aprendizagem é facilitada por interações sociais: proporcionar oportunidades para os estudantes articularem suas ideias e seus entendimentos para os colegas, assim como para ouvirem e discutirem as ideias dos outros no contexto da sala de aula é particularmente efetivo para aumentar a aprendizagem conceitual. Não por mero acaso, essa é uma das estratégias básicas pesquisadas e sugeridas por Mazur e seu grupo"[1]. Além disso, promover o acesso dos estudantes da rede pública aos centros de ciências, proporcionando uma vivência em outras formas de educação.

A proposta pretende enriquecer a abordagem dos conteúdos astronômicos importantes para a formação do aluno. Um deles é a cinemática do movimento, que será aplicada ao movimento dos planetas. Utilizando gráficos da órbita, velocidade e aceleração, conseguimos visualizar seu comportamento, favorecendo um entendimento mais completo, sendo possível mostrar, em tempo real, os efeitos da mudança da velocidade inicial na trajetória descrita pelo planeta (circular, elíptica ou parabólica).

Essa proposta enfatiza a relevância que deve ser dada às atividades que possibilitem o aumento do capital cultural dos alunos, sobretudo no estudo de temas científicos. Finalizando todas as etapas da proposta foi aberto um espaço para que eles colocassem suas opiniões e dúvidas sobre o tema, seguido da implementação do segundo questionário (questionário de avaliação de resultados). No Apêndice B estão apresentados os questionários e o resultado da avalição para uma amostragem de alunos em uma turma.

Como pode ser constatada, pela comparação dos resultados dos questionários apresentados no apêndice-B, a proposta de atividades oferecidas serviu efetivamente tanto para incentivar o uso de práticas didáticas diferenciadas daquelas utilizadas na educação formal, no sentido de ampliar e fortalecer sua formação acadêmica (conteúdo) dos currículos escolares. Além disso, mostrou que a utilização de novos recursos didáticos na prática docente, permite a maior motivação para introdução de temas da atualidade no ensino de Física.

\section{Material Suplementar}

O material suplementar a seguir está disponível online: Apêndice A

Apêndice B

\section{Referências}

[1] C.A.C.M. Dias e Josué R. Santa Rita, Revista LatinoAmericana de Educação em Astronomia 6, 55 (2008).

[2] O. Borges, Revista Brasileira de Ensino de Física 28, 135 (2006).

[3] M.A. Moreira, Ensino de Física no Brasil, Retrospectiva (Instituto de Física, UFRGS, 2000).

[4] C.L.A. Ladera and P. Eduardo e Léon, Latin-American Journal of Physics Education 4, 2 (2010).
[5] F. Zwicky, PNAS 15, 773 (1929).

[6] L. Sodre Jr., Caderno Brasileiro Ensino Física 27, 743 (2010).

[7] C.A Wuensche. A Radiação Cósmica de Fundo em Microondas e a Formação de Estruturas no Universo: Uma Visão Atual (INPE, São José dos Campos, 1994). 